Hor monal regul at $i$ on of the death commit ment in progr ammed cel I death of the si I kwormant er $\mathrm{i}$ or si I k gl ands

\begin{tabular}{|l|l|}
\hline 著者 & $\begin{array}{l}\text { Nat sui } \mathrm{H} \text { r ot o, Kakei Not onor i , I wam Nasaf um , } \\
\text { Sakur ai Sho }\end{array}$ \\
\hline $\begin{array}{l}\text { j our nal or } \\
\text { publ i cat i on ti t l e }\end{array}$ & Journal of I nsect Physi ol ogy \\
\hline vol une & 58 \\
\hline number & 12 \\
\hline page r ange & $1575-1581$ \\
\hline year & $2012-12-01$ \\
\hline URL & ht t p: //hdl . handl e. net /2297/33402 \\
\hline
\end{tabular}




\section{Hormonal regulation of the death commitment in programmed cell death of the silkworm anterior silk glands}

Hiroto Matsui ${ }^{1,3}$, Motonori Kakei ${ }^{2,4}$, Masafumi Iwami ${ }^{1,2}$, Sho Sakurai ${ }^{1,2}$

${ }^{1}$ Division of Biological Sciences; 2 Division of Life Sciences, Graduate School of Natural Science and Technology, Kanazawa University, Kanazawa 920-1192, Japan

${ }^{3}$ Present address: Biological Research Laboratories, Nissan Chemical Industries, Shiraoka, Shiraokamachi, Saitama, 349-0294, Japan

${ }^{4}$ Present address: Translational Medical Center, National Center of Neurology and Psychiatry, Ogawahigashimachi, Kodaira, Tokyo 187-8551, Japan

Corresponding author

Sho Sakurai, Division of Life Sciences, Graduate School of Natural Science and Technology, Kanazawa University, Kanazawa 920-1192.

E-mail:ssakurai@staff.kanazawa-u.ac.jp

Phone: +81 762645083

Fax: +81 762344017 


\section{Abstract}

During larval-pupal transformation, the anterior silk glands (ASGs) of the silkworm Bombyx mori undergo programmed cell death (PCD) triggered by 20-hydroxyecdysone (20E). Under standard in vitro culture conditions $(0.3 \mathrm{ml}$ of medium with $1 \mu \mathrm{M} 20 \mathrm{E}$ ), ASGs of the fourth-instar larvae do not undergo PCD in response to 20E. Similarly, larvae of the fifth instar do not respond to $20 \mathrm{E}$ through day 5 of the instar (V5). However, ASGs of V6 die when challenged by 20E, indicating that the glands might be destined to die before V6 but that a death commitment is not yet present. When we increased the volume of culture medium for one gland from $0.3 \mathrm{ml}$ to $9 \mathrm{ml}$, V5 ASGs underwent PCD. We examined the response of ASGs to 20E every day by culturing them in $9 \mathrm{ml}$ of medium and found that ASGs on and after V2 were fully responsive to $20 \mathrm{E}$. Because pupal commitment is associated with juvenile hormone (JH), the corpora allata (a JH secretory organ) were removed on day 3 of the fourth larval instar (IV3), and their ASGs on V0 were cultured with 20E. Removal of the corpora allata allowed the V0 larval ASGs to respond to 20E with PCD. In contrast, topical application of a $\mathrm{JH}$ analogue inhibited the response to $20 \mathrm{E}$ when applied on or before V5. We conclude that the acquisition of responsiveness to $20 \mathrm{E}$ precedes the loss of $\mathrm{JH}$ sensitivity, and that the death commitment in ASGs occurs between V5 and 6.

Key words: anterior silk gland; Bombyx mori; commitment; ecdysone; juvenile hormone; programmed cell death; glucose oxidase; hydrogen peroxide 


\section{Introduction}

In insects, the larval-specific tissues are partly or entirely eliminated from the pupal bodies during larval-pupal transformation in response to a metamorphic increase in hemolymph ecdysteroid concentration (von Gaudecker and Schmale, 1974; Chinzei, 1975; Schwartz, 1992; Terashima et al., 2000). In addition, larval tissues respond to 20-hydroxyecdysone (20E), a biologically active type of ecdysteroid, by undergoing various developmental changes. These changes are determined in advance according to the actual responses, and this process is known as pupal commitment (Riddiford, 1985).

Bombyx mori anterior silk gland (ASG) is a larval-specific tissue that is eliminated through programmed cell death (PCD) in response to 20E. This PCD occurs after the onset of cocoon spinning during the latter part of day 5 of the fifth larval instar (V5) (Terashima et al., 2000). Similarly, ASGs after the onset of spinning undergo PCD when treated with $20 \mathrm{E}$ in vitro. The ability of ASGs to respond to 20E first appears in some ASGs late V5 and in all on V6, the day of wandering (Kakei et al., 2005).

In pupal commitment, acquisition of responsiveness to $20 \mathrm{E}$ is the first step of the change in commitment. $\mathrm{JH}$ inhibits this change in commitment, and the commitment is completed by the loss of sensitivity to JH (Riddiford, 1985; Obara et al., 2002; Koyama et al., 2004). In B. mori fifth instar ASGs, the loss of sensitivity to $\mathrm{JH}$ begins between V4 and V5 of the instar and is completed by V6 (Kakei et al., 2005). However, the ASGs first exhibit responsiveness to 20E late on V5, which is after the beginning of the loss of sensitivity to JH. These observations led us to question whether the commitment to death occurs in ASGs in a manner 
similar to that observed in epidermis and imaginal discs.

$20 \mathrm{E}$ initially binds to ecdysone receptor $(\mathrm{EcR})$ and then sequentially regulates early-response gene expression. This mode of action is referred to as a genomic action of a steroid hormone. In the 20E-induced PCD of the Drosophila melanogaster salivary gland, the action of $20 \mathrm{E}$ begins with a hierarchical regulation of early-response genes and culminates with the activation of late-response genes. A similar hierarchical expression of early-response genes occurs in the 20E-induced PCD of B. mori ASGs (Sekimoto et al., 2006, 2007).

In addition to genomic action, 20E exhibits non-genomic action. Specifically, its downstream effects activate the death effector caspase 3-like protease (Iga et al., 2010). Indirect evidence indicates that the non-genomic action begins with $20 \mathrm{E}$ binding to a putative membrane ecdysone receptor, which likely belongs to a family of G-protein-coupled receptor, and activating a signal transduction pathway. This action is followed by the activation of caspase 3-like protease, which completes the PCD via DNA fragmentation (Manaboon et al., 2009).

We recently identified a third factor in the control of 20E-induced PCD; this factor appears in the medium during ASG culture and inhibits the action of $20 \mathrm{E}$ (Kakei et al., 2005). This PCD inhibitory factor was identified as glucose oxidase (GOD), which may be produced by ASG cells. The catalytic by-product of GOD, hydrogen peroxide $\left(\mathrm{H}_{2} \mathrm{O}_{2}\right)$, is the immediate inhibitory factor (Matsui et al., 2011).

ASGs in vitro do not respond to 20E by undergoing PCD before the onset of spinning. However, this lack of response to $20 \mathrm{E}$ is not caused by the glands' own lack of competency to respond to 20E. Rather, the lack of response is a result of the $\mathrm{H}_{2} \mathrm{O}_{2}$ that is produced by the GOD released into the medium. In addition, the 
production of $\mathrm{H}_{2} \mathrm{O}_{2}$ in the medium conceals the involvement of $\mathrm{JH}$ in the acquisition of responsiveness to $20 \mathrm{E}$. These multiple factors make it difficult to examine whether ASGs are committed to die (death commitment) before the spinning (Kakei et al., 2005).

We therefore developed a simple method to limit the effects of GOD on the ASGs. Specifically, the ASGs were incubated in a large volume of culture medium, which reduced the $\mathrm{H}_{2} \mathrm{O}_{2}$ concentration to a level at which it had no effect on the ASGs. Using this culture condition, we examined the presence of the death commitment and its timing. At V2 (4 days before the onset of spinning), B. mori ASGs respond to 20E by undergoing cell death. At the onset of spinning (late V5 early V6), however, the glands completely lose their sensitivity to JH. After V2, the $\mathrm{JH}$ concentration in the hemolymph is very low, but the glands are regulated not to respond to 20E. These unstable conditions between V2 and V6 are strictly controlled by GOD, which blocks the cell death pathway if ASGs are exposed to high 20E. This study is the first to show the presence of the death commitment in in insect metamorphosis and proposes a unique control mechanism for cell death.

\section{Materials and Methods}

\subsection{Animals}

Larvae of the silkworm B. mori were reared and staged as previously described (Sakurai et al., 1998). Newly molted fifth instar larvae were fed from the beginning of the photophase following the scotophase during which they molted to fifth instars. The $24 \mathrm{~h}$ period of the photophase following the scotophase during which the fourth-instar larva molted was designated day 0 of the fifth instar (V0). 
ASGs were dissected during the photophase of each day. Corpora allata (CA) were removed from fourth-instar larvae as described (Sakurai, 1983).

\subsection{Hormones and tissue culture}

A juvenile hormone analogue (JHA), S-methoprene (95\% stereochemically pure; SDS Biotech, Tokyo), was dissolved in acetone, and a 5- $\mu$ l aliquot was applied to the dorsal surface of individual larvae. 20E (Sigma, St. Louis, MO) was dissolved in distilled water $(1 \mathrm{mg} / \mathrm{ml})$ and stored at $-20^{\circ} \mathrm{C}$. ASGs were rinsed with Grace's insect cell culture medium (Gibco BRL, Rockville, MD) and cultured individually in Grace's medium ( $\mathrm{pH}$ 6.4, adjusted with $\mathrm{NaOH}$ ) with or without $1 \mu \mathrm{M} 20 \mathrm{E}$ at $25^{\circ} \mathrm{C}$. The ASGs were observed every $24 \mathrm{~h}$, and the degree of PCD progression was noted with PCD score according to the changes in their cellular morphology (Terashima et al., 2000; Kakei et al., 2005), where a score of 0 indicates no change, and a score of 6 indicates the completion of PCD.

\subsection{Staining}

ASGs were fixed with $4 \%$ formaldehyde for more than 30 min and washed with phosphate-buffered saline (PBS: $137 \mathrm{mM} \mathrm{NaCl}, 2.7 \mathrm{mM} \mathrm{KCl}, 8.1 \mathrm{mM} \mathrm{Na} 2 \mathrm{HPO}_{4}$, $1.47 \mathrm{mM} \mathrm{KH}_{2} \mathrm{PO}_{4}, \mathrm{pH}$ 7.4). The samples were then incubated in PBS containing DAPI $(0.1 \mu \mathrm{g} / \mathrm{ml})$ at $25^{\circ} \mathrm{C}$ in the dark for $15 \mathrm{~min}$. Finally, the samples were washed with PBS and observed under a fluorescence microscope using a UV excitation filter (BX-50, Olympus, Tokyo).

\subsection{Reverse transcription (RT)-PCR}


Total RNA was extracted from ASGs (Chomcyzuski and Sacchi, 1987) and treated with RNase-free DNase (Promega, Madison, WI). Complementary DNA (cDNA) was prepared from $1 \mu \mathrm{g}$ of total RNA using anchored oligo-dT [5'-(T)12(A/C/G)(A/C/G/T)-3'] and Rever'Tra Ace reverse transcriptase (Toyobo, Osaka, Japan). For RT-PCR, Krüppel homolog $1(K r-h 1)$ cDNA was amplified for 40 cycles with the following primers: forward, 5'-GCGAGTGTGGTTTGACATTG; reverse, 5'-GATACGGCCTCTCCTTTGTG. RT-PCR targeting 20E-induced genes was performed using the same primer sets as described in Sekimoto et al., 2006. RNA encoding ribosomal protein L3 $(R p L 3)$ was used as an internal standard and amplified for 25 cycles. PCR products were separated by agarose gel electrophoresis.

\subsection{DNA isolation and agarose gel electrophoresis}

ASGs were homogenized and mixed in DNA extract buffer (10 mM Tris-HCl, 150 $\mathrm{mM} \mathrm{NaCl}, 10 \mathrm{mM}$ EDTA-NaOH, 0.1\% sodium dodecylsulfate, $\mathrm{pH}$ 8.0) on ice. The homogenate was treated with RNase $\left(20 \mu \mathrm{g} / \mathrm{ml}, 37^{\circ} \mathrm{C}, 30 \mathrm{~min}\right)$ and proteinase $\mathrm{K}$ $\left(100 \mu \mathrm{g} / \mathrm{ml}, \quad 50^{\circ} \mathrm{C}, \quad 60 \mathrm{~min}\right)$. DNA was extracted using a standard phenol-chloroform and chloroform extraction method. The DNA was then electrophoresed on a $2 \%$ agarose gel in TAE buffer and stained with ethidium bromide.

\subsection{Hydrogen peroxide determination}

The concentration of hydrogen peroxide was determined by measuring the change in absorbance of the reaction mixture at $500 \mathrm{~nm}$. A total volume of $1 \mathrm{ml}$ of the 
reaction mixture consisted of $0.17 \mathrm{mM}$ o-dianisidine- $\mathrm{HCl}$ (Sigma) in $20 \mathrm{mM} \mathrm{PB}$ (24.4 mM Na $\mathrm{HPO}_{4}$ and $15.6 \mathrm{mM} \mathrm{NaH} \mathrm{PO}_{4}, \mathrm{pH}$ 7.0), $60 \mathrm{U} / \mathrm{ml}$ horseradish peroxidase (Sigma), and $34.5 \mu \mathrm{l}$ of a sample taken from the culture medium. For the negative control, $34.5 \mu \mathrm{l}$ of $20 \mathrm{mM}$ PB was added instead of the sample. The samples were added and incubated at $35^{\circ} \mathrm{C}$ for $5 \mathrm{~min}$, and then, the absorbance at $500 \mathrm{~nm}$ was recorded.

\section{Results}

\section{1. ASG culture in extra volumes of Grace's medium}

During the culture of V3 ASGs in Grace's medium, the ASGs generated GOD (Matsui et al., 2011). The GOD utilized glucose in the medium as a substrate to produce $\mathrm{H}_{2} \mathrm{O}_{2}$. This $\mathrm{H}_{2} \mathrm{O}_{2}$ inhibited V3 ASGs from responding to 20E with PCD. This inhibitory effect made it difficult to determine the time at which ASGs became competent to respond to 20E. V3 ASGs cultured in Ringer's solution died due to a lack of glucose in the solution; thus, no $\mathrm{H}_{2} \mathrm{O}_{2}$ was generated by these ASGs. The V3 ASGs survived when the Ringer's solution was supplemented with $0.01 \% \mathrm{H}_{2} \mathrm{O}_{2}$ (approximately $3 \mathrm{mM}$ ) but died at $0.005 \% \mathrm{H}_{2} \mathrm{O}_{2}$. These results indicate that a minimum concentration of $\mathrm{H}_{2} \mathrm{O}_{2}$ in the medium was crucial to inhibit the action of $20 \mathrm{E}$. Thus, the ASGs were cultured in various volumes of the medium to reduce the GOD concentration and thereby reduce the $\mathrm{H}_{2} \mathrm{O}_{2}$ concentration to reduce its inhibitory effects. We cultured V3 ASGs in the medium at volumes equal to 10,20 or 30 times higher than the standard culture volume of $0.3 \mathrm{ml}$ (Fig. 1). V3 ASGs survived when cultured in 0.3 or $3 \mathrm{ml}$ of medium. In $6 \mathrm{ml}$, however, the ASGs showed some but not all of the morphological changes 
associated with PCD. In $9 \mathrm{ml}$ of medium, the ASGs underwent cell death with a PCD score of $4.8 \pm 1.1$ (Fig. 1A). These values indicated that the V3 ASGs were sensitive to $20 \mathrm{E}$, but the $\mathrm{GOD} / \mathrm{H}_{2} \mathrm{O}_{2}$ may have suppressed the progress of PCD.

To test this hypothesis, we measured the changes in $\mathrm{H}_{2} \mathrm{O}_{2}$ concentration during culture in $0.3,3,6$ or $9 \mathrm{ml}$ of medium (Fig. 1B-E). When an ASG was cultured in 9 $\mathrm{ml}$ of medium, the $\mathrm{H}_{2} \mathrm{O}_{2}$ concentration at $72 \mathrm{~h}$ was $0.31 \pm 0.071 \mathrm{mM}$. In contrast, during culture in $0.3 \mathrm{ml}$ of medium, the $\mathrm{H}_{2} \mathrm{O}_{2}$ concentration was $0.625 \pm 0.107 \mathrm{mM}$ at $3 \mathrm{~h}$ (Fig. 1B). Accordingly, an increase in the medium volume resulted in the death of the V3 ASGs. The death of the ASGs was a result of the reduction of the $\mathrm{H}_{2} \mathrm{O}_{2}$ concentration below an inhibitory level. Therefore, we used $9 \mathrm{ml}$ of the medium in the experiments described below.

\subsection{The timing of the acquisition of $20 \mathrm{E}$ responsiveness}

The time when ASGs become responsive to $20 \mathrm{E}$ was determined by culturing the ASGs every day from IV4 to V7 in $9 \mathrm{ml}$ of medium with $20 \mathrm{E}$ for $144 \mathrm{~h}$. The majority of IV4, V0 and V1 ASGs exhibited the type B form (Fig. 4A; see Kakei et al., 2005 for type B form). Specifically, the cells exhibited blebbing, the nuclear morphology at $144 \mathrm{~h}$ showed small amounts of cell and nuclear condensation (Fig. 2D, d). The PCD scores of the others were below 3. In contrast, when the V2 ASGs were cultured in $9 \mathrm{ml}$ of the medium with $20 \mathrm{E}$, no type B forms were observed. Instead, approximately $40 \%$ of the ASGs completed PCD (Fig. $2 \mathrm{~F}$, f) and had PCD scores of 6. Most of the remaining ASGs attained PCD scores of 5 (average PCD score, $4.9 \pm 1.0$; Fig. 2A). In these ASG cells, DNA fragmentation occurred (Fig. 2B, f), but not in the V1 cells (Fig. 2B). These results indicate that the V2 ASGs were 
competent to respond to $20 \mathrm{E}$ by completing PCD.

To examine whether the acquisition of responsiveness to $20 \mathrm{E}$ is associated with specific gene expression changes, the expression of early and early-late genes was determined for V1 and V2 ASGs (Fig. 2G). Among the genes examined, the expression levels of five genes, $E c R-B 1$ (ecdysone receptor-B1), E75A, $E 75 B$, BFTZ-F1 and BHR3 (Bombyx hormone receptor 3), were equally enhanced in response to $20 \mathrm{E}$ in both ASGs. This result indicates that those genes may not be involved in the change in the responsiveness. Among the 13 genes examined, only the level of $E 74 B$ exhibited a difference between the V1 and V2 ASGs in the absence as well as in the presence of $20 \mathrm{E}$. The $E 74 B$ basal expression (in the absence of 20E) was almost undetectable on V1 but became detectable on V2. Moreover, 20E greatly enhanced the expression of E74B in V2 ASGs, but only slightly increased its expression in V1 ASGs. Among the three $B R-C$ isoforms, only the Z1 isoform was expressed in those ASGs. 20E exerted no effects on Z1 isoform mRNA expression in the V1 ASGs but increased it in the V2 ASGs. However, the basal expression level of the $\mathrm{Z} 1$ isoform decreased from V1 to V2, indicating that the decrease could be important to the acquisition of competence to respond to $20 \mathrm{E}$.

\subsection{Suppression of the change in the responsiveness to $20 E$}

The results above show that V2 ASGs are competent to respond to $20 \mathrm{E}$ by undergoing cell death. If this early phenomenon were associated with the change in commitment to die, $\mathrm{JH}$ should affect the responsiveness to $20 \mathrm{E}$. To investigate this issue, CA were removed from IV3 larvae, and JHA or acetone was topically 
applied immediately after the removal. The ASGs of the larvae that successfully molted into the fifth instar were cultured in $9 \mathrm{ml}$ of the medium with $20 \mathrm{E}$ on V0 and V3. The ASGs of allatectomized V0 larvae completed PCD in $83 \%$ of the glands, while those that were treated with JHA did not undergo PCD. Specifically, the PCD score was 3 in approximately 30\% of the JHA-treated glands, and the remaining glands exhibited the type B morphology that showed no nuclear fragmentation (Fig. 3A), which was similar to the response to 20E of the intact V1 larval ASGs. Similar results were obtained for the ASGs that were examined on V3 (Fig. 3B), although the intact V3 larval ASGs underwent PCD under the same culture conditions. These results indicate that JHA is involved in the acquisition of responsiveness to $20 \mathrm{E}$.

\subsection{The timing of the loss of sensitivity to $\mathrm{JH}$}

To determine the time when ASGs lose their sensitivity to JH, fifth instar larvae were treated with JHA, and the responsiveness of ASGs of those larvae were examined in vitro two days later (Fig. 4A). The gland response to $20 \mathrm{E}$ was suppressed when JHA was applied on or before V4. In those ASGs, 40-90\% of the glands exhibited the type B form, and the remaining glands had PCD scores below 3. In larvae treated with JHA on V5, approximately half of the ASGs underwent PCD (PCD scores of 5 or 6), but the other half of the ASGs exhibited responses similar to the glands of larvae treated with JHA before V5. V6 ASGs exhibited a complete response to 20E, which demonstrates that the ASGs began to lose sensitivity to JH around V5 and completely lost it between V5 and V6.

To examine the time when the ASGs become insensitive to JHA, we applied 
JHA on each day of the fifth instar, and examined the gland responses to $20 \mathrm{E}$ in vitro on V8.. Figure 5 shows that the JHA applied on V2-V5 was effective at preventing most of the $20 \mathrm{E}$-induced cell death in the glands. The inhibitory effect of applying JHA on V2 lasted until V8, at which time the gland's response was examined. When given JHA on V5 and assayed on V8, 42\% exibited the Type B form and the remaining ASGs scored 2-3 in PCD, whereas in the larvae allatectomized on IV3, the ASGs treated with JHA at the same time and assayed two days later showed more extensive PCD (Fig. 4A). These results indicate that the V5 ASGs were less responsive to 20E than those shown in Fig. 4.

\subsection{Kr-h1 expression and JHA}

In the intracellular signaling pathway of $\mathrm{JH}$, methoprene-tolerant protein (Met) acts as a JH receptor, and Krüppel homolog 1 (Kr-h1) plays a key role in the hierarchical control of the expression of Met downstream target genes (Minakuchi et al., 2008; Konopova et al., 2011). To examine whether the loss of sensitivity to $\mathrm{JH}$ is associated with $K r-h 1$ expression, we measured $K r-h 1$ expression by RT-PCR. In intact larval ASGs, $K r-h 1$ expression was high on V0, low but detectable on V1, and very low on V2 (Fig. 3C, left panel). V0 larvae were topically treated with JHA or acetone, and their ASGs were examined on V3. The $K r-h 1$ expression in the acetone-treated ASGs was very low on V3 (similar to intact larval ASGs), while in the JHA treatment group, the expression level was as high as in V0 ASGs. Accordingly, $K r-h 1$ expression appeared to be retained in the presence of $\mathrm{JH}$ (Fig. 3C, right panel) irrespective of the larval age.

JHA treatment enhanced $K r-h 1$ expression irrespective of the acquisition of responsiveness to 20E (Fig. 4). Larvae were allatectomized on IV3, and JHA was 
applied on V4, 5 or 6. The expression level was examined 2 days later (Fig. 4B). A single application of JHA greatly increased $K r-h 1$ expression irrespective of the day of JHA application, showing that $K r-h 1$ expression depends on the presence of JHA.

\section{Discussion}

A series of changes in the tissue responses to $20 \mathrm{E}$ and $\mathrm{JH}$ is an indication of the change in commitment (Riddiford, 1985); the acquisition of responsiveness to $20 \mathrm{E}$ precedes to the loss of sensitivity to JH (Riddiford, 1985 for epidermis, Obara et al., 2002 for wing discs). Tissues such as epidermis and imaginal discs are pupally committed during feeding period of the last-larval instar and undergo morphogenetic changes to form pupal tissues during pupal metamorphosis. In contrast, it remained unclear whether larval-specific tissues that are eliminated from insect bodies are destined to die and whether there is a death commitment occurring in the larval period. Using B. mori ASGs, we were able to partially answer these questions.

The B. mori ASGs became responsive to 20E by undergoing PCD on V2, and they lost sensitivity to JH on V6 (the end of the feeding period) (Fig. 6). The ASGs progress to death through a series of steps. 20E did not induce PCD in V1 ASGs even when they were cultured in $9 \mathrm{ml}$ of medium, but V2 ASGs underwent PCD when cultured under the same conditions. This result clearly shows that ASGs became responsive to $20 \mathrm{E}$ between $\mathrm{V} 1$ and V2. The acquisition of responsiveness required a disappearance of $\mathrm{JH}$ from the larvae. When the CA were removed on IV3, the ASGs of V0 larvae underwent PCD, while the V0 intact larval ASGs 
never did so. Alternatively, topical application of JHA to intact larvae on V0 suppressed the acquisition of responsiveness to $20 \mathrm{E}$. These results indicate that maintaining JH in ASGs led to inhibition of responsiveness to 20E.

Our previous study suggested that the sensitivity to JH may be lost between V4 and V5 (Kakei et al., 2005). The present study clarified the timing of the loss of sensitivity. JHA application on V4 prevented the ASGs from undergoing PCD by $20 \mathrm{E}$, but the application on V5 failed to inhibit the $20 \mathrm{E}$ response in one-third of the ASGs examined. In addition, JHA applied on V5 did not inhibit the 20E-induced PCD to any degree. Thus, the ASGs may begin to lose their sensitivity between V4 and V5 and lose it entirely by V6. Because it is recognized that the pupal commitment is completed when a given tissue loses its sensitivity to JH (Riddiford, 1985), the death commitment in ASGs may be completed on V6.

Prior to V6, ASGs do not undergo PCD under standard culture conditions (Matsui et al., 2011). The concentration of JH in the hemolymph is very low between V2 (the day of acquisition of responsiveness to 20E) and V6 (the loss of sensitivity to JH) (Sakurai and Niimi, 1997), indicating that JH may not be involved in the lack of response to $20 \mathrm{E}$ during this period. $\mathrm{GOD} / \mathrm{H}_{2} \mathrm{O}_{2}$ in ASGs inhibits the gland response to 20E (Matsui et al., 2011), and in the ASGs between V2 and V5, GOD may play a critical role in inhibiting PCD. The critical concentration of $20 \mathrm{E}$ to induce PCD is $1 \mu \mathrm{M}$ (Terashima et al., 2000). Although this concentration has not been recorded during the feeding period of any lepidopteran larvae (Dean et al., 1980; Koyama et al., 2004), GOD may serve as a biochemical assurance that ASGs never undergo PCD in response to an unexpected rise in hemolymph ecdysteroid levels during the feeding period. GOD 
in the ASG inner cavity is discarded with released silk proteins in the early phase of cocooning. After discarding GOD, the ASGs are allowed to respond to $20 \mathrm{E}$. Consequently, they are eliminated through PCD, which is triggered by a metamorphic rise in hemolymph ecdysteroid concentration. Thus, the 20E-induced PCD of ASGs is controlled by a stage-specific dual regulation.

The dual regulation in $B$. mori ASGs is unique among animal tissues that are eliminated at metamorphosis. In amphibians, most tissues of the tadpole undergo a complete transformation from larval to adult stage. Thyroid hormone (TH) plays a critical role in the degeneration of larval tissues and the concurrent proliferation and differentiation of adult tissues in a developmental stage-dependent manner. The elimination of the larval tail is largely achieved through cell apoptosis during the climax (NF stage 58-65), at which point $\mathrm{TH}$ levels are at their maximum. Tails isolated from tadpoles as late as stage 49 do not respond to high concentrations of $\mathrm{TH}$, while those from stage 51 can be induced to undergo resorption (Shaffer, 1963). At early stages of metamorphosis, the larval tails express low levels of the gene encoding the TH-inactivating enzyme type III deiodinase (D3) (Kawahara et al., 1999). At the onset of tail resorption, D3 expression declines, and the gene encoding type II deiodinase (D2) is upregulated. D2 catalyzes the deiodination of the outer ring of T4, which results in a more potent thyroid hormone, T3 (Cai and Brown, 2004). In tails, TR $\alpha$ and TR $\beta$ mRNA levels rise in parallel with an increase in endogenous $\mathrm{TH}$ concentration (Wang and Brown, 1993), and adequate receptor expression is necessary to obtain tissue sensitivity to TH (Wang and Brown, 1993). Thus, the timing of death in amphibian tails may be regulated primarily by $\mathrm{TH}$ and 
secondarily by the expression of other genes, such as $D 2, D 3$ and $T R$, which may be influenced by the changing $\mathrm{TH}$ concentrations.

In ASGs, the expression levels of EcR and EcR partner protein, Ultraspiracle (Usp), do not change remarkably from V1 to V2 (Kaneko et al., 2006). This lack of change indicates a lack of relationship between $E c R$ expression and the acquisition of responsiveness to $20 \mathrm{E}$, in contrast to the dynamic of $\mathrm{TH}$ receptors in amphibian tails. Culturing V2 ASGs in $9 \mathrm{ml}$ of the medium with $20 \mathrm{E}$ induced DNA fragmentation, but this was not the case for V1 ASGs. This result indicates that the $20 \mathrm{E}$ signaling pathway must be completed to the point of activation of caspase 3-like protease (Iga et al., 2010) in V2 ASGs but not in V1 glands. Accordingly, some factor(s) in the pathway must be up- or down-regulated between V1 and V2, but we could not find any early genes with notable differences in expression between V1 and V2 ASGs.

JH application before V5 inhibited the execution of PCD; the PCD scores remained less than 4, but type B forms appeared in 40-90\% of specimens. The type B form is characterized by the appearance of plasma membrane blebbing, cell shrinkage and nuclear condensation, but nuclear and DNA fragmentation do not occur in these cells (Fig. 2; Kakei et al., 2005). Nuclear and DNA fragmentation are under the control of the PKC-caspase 3 pathway (Iga et al., 2010), while the pathway up to nuclear condensation is likely mediated by the CaMK pathway (Manaboon, 2009) and cell shrinkage is under the genomic pathway (Iga et al., 2007). Accordingly, the present results indicate that $\mathrm{JH}$ may inhibit the non-genomic pathway up to caspase 3-like protease activation leading to DNA and nuclear fragmentation. The final process of apoptosis, which is associated with 
nuclear and DNA fragmentation by activated caspase 3 -like protease, is under the developmental regulation of the death commitment occurring on V6. Since the type B form occurs in ASGs before V2, ASGs are competent to respond to 20E in vitro by exhibiting nuclear condensation and cell shrinkage. These phenomena do not occur in vivo at the end of the fourth instar, when ASGs experience a peak hemolymph ecdysteroid concentration of approximately $1 \mu \mathrm{M}$ (Koyama et al., 2004), and the mechanism of inhibition of apoptosis at the time of larval-larval molting remains unknown.

$\mathrm{JH}$ controls gene expression through its putative receptor Met and Kr-h1 that is an early JH-response gene located downstream of Met (Minakuchi et al., 2008, 2009; Liu et al., 2009; Konopova et al., 2011; Lozano and Belles, 2011). In the D. melanogaster fat body, JH counteracts Met to prevent caspase-dependent PCD and thereby controls fat body remodeling and larval-pupal metamorphosis. In $B$. mori, $K r^{-h} 1$ expression declined to very low level on $\mathrm{V} 2$, when the in vivo JH titer is very low (Sakurai and Niimi, 1997). A single application of JHA, however, enhanced $K r-h 1$ expression irrespective of the time of JH application. This effect was observed even on V6, when the ASGs were committed to die and not responsive to JHA, indicating that $\mathrm{Kr}$-h1 is not involved in the change in commitment. Also, Kr-h1 may not mediate the JHA counteraction to inhibit 20E-induced DNA fragmentation, because JHA application on V6 did not inhibit the 20E-induced PCD, while inducing high $K r$-h1 expression (present results, Kakei et al., 2005). Moreover, these observations suggest that, in this case, JH action is mediated by an alternative signaling pathway that does not use $\mathrm{Kr}$-h1 as a transducer. 


\section{Acknowledgments}

This work was supported by the JSPS Grant-in-Aid for Scientific Research 21380035 (to S.S.). 


\section{References}

Cai, L., Brown, D.D., 2004. Expression of type II iodothyronine deiodinase marks the time that a tissue responds to thyroid hormone-induced metamorphosis in Xenopus laevis. Developmental Biology 266, 87-95.

Chinzei, Y., 1975. Induction of histolysis by ecdysterone in vitro: breakdown of anterior silk gland in silkworm Bombyx mori (Lepidoptera: Bombycidae). Applied entomology and zoology 10, 136-138.

Chomcyznski, P., Sacchi, N., 1987. Single step method of RNA isolation by acid guanidinium thiocyanate-phenol-chloroform extraction. Analytical Biochemistry 162: 156-159.

Dean, R.L., Bollenbacher, W.E., Locke, M., Smith, S.L., Gilbert, L.I., 1980. Haemolymph ecdysteroid levels and cellular events in the intermoult/moult sequence of Calpodes ethlius. Journal of Insect Physiology 26, 267-280.

Iga, M., Manaboon, M., Matsui, H., Sakurai, S., 2010. Ca ${ }^{2+-P K C-c a s p a s e ~ 3-l i k e ~}$ protease pathway mediates DNA and nuclear fragmentation in ecdysteroid-induced programmed cell death. Molecular and Cellular Endocrinology 321, 146-151.

Kakei, M., Iwami, M., Sakurai, S., 2005. Death commitment in the anterior silk gland of the silkworm, Bombyx mori. Journal of Insect Physiology 51, 17-25.

Kawahara, A., Gohda, Y., Hikosaka, A., 1999. Role of type III iodothyronine 5-deiodinase gene expression in temporal regulation of Xenopus metamorphosis. Development, growth \& differentiation 41, 365-373.

Kaneko, Y., Takaki, K., Iwami, M., Sakurai, S., 2006. Developmental profile of annexin IX and its possible role in programmed cell death of the Bombyx mori 
anterior silk gland. Zoological Science 23, 533-542.

Konopova, B., Smykal, V., Jindra, M., 2011. Common and distinct roles of juvenile hormone signaling genes in metamorphosis of holometabolous and hemimetabolous insects. PloS One 6, e28728.

Koyama, T., Obara, Y., Iwami, M., Sakurai, S., 2004. Commencement of pual commitment in late penultimate instar and its hormonal control in wing imaginal discs of the silkworm, Bombyx mori. Journal of Insect Physiology 50, $123-133$.

Liu, Y., Sheng, Z., Liu, H., Wen, D., He, Q., Wang, S., Shao, W., Jiang, R-J., An, S., Sun, Y., Bendena, W. G., Wang, J., Gilbert, L. I., Wilson, T. G., Song, Q., Li, S., 2009. Juvenile hormone counteracts the bHLH-PAS transcription factors MET and GCE to prevent caspase-dependent programmed cell death in Drosophila. Development 136, 2015-2025.

Lozano, J. and Belles, X., 2011. Conserved repressive function of Krüppel homolog 1 on insect metamorphosis in hemimetabolous and holometabolous species. Scientific Reports 1, 163.

Manaboon, M., Iga, M., Iwami, M., Sakurai, S., 2009. Intracellular mobilization of $\mathrm{Ca}^{2+}$ by the insect steroid hormone 20 -hydroxyecdysone during programmed cell death in silkworm anterior silk glands. Journal of Insect Physiology 55, 122-128. Matsui, H., Kakei, M., Iwami, M., Sakurai, S., 2011. Glucose oxidase prevents programmed cell death of the silkworm anterior silk gland through hydrogen peroxide production. FEBS Journal 278, 776-785.

Minakuchi, C., Zhu, X., Riddiford, L. M., 2008. Krüppel homolog 1 (Kr-h1) mediates juvenile hormone action during metamorphosis of Drosophila 
melanogaster, Mechanism Development 125, 91-105.

Minakuchi, C., Namiki, T., Shinoda, T., 2009. Krüppel homolog 1, an early juvenile hormone-response gene downstream of Methoprene-tolerant mediates its anti-metamorphic action in the red flour beetle Tribolium castaneum. Developmental Biology 325, 341-350.

Obara, Y., Miyatani, M., Ishiguro, Y., Hirota, K., Koyama, T., Izumi, S., Iwami, M., Sakurai, S., 2002. Pupal commitment and its hormonal control in wing imaginal discs. Journal of Insect Physiology 48, 933-944.

Riddiford, L. M., 1985. Hormone action at the cellular level. In: Kerkut, G. A., Gilbert, L. I. (Eds), Comprehensive insect physiology, biochemistry, and pharmacology, Vol. 8. Pergamon Press, New York, pp. 37-84.

Sakurai, S., 1983. Temporal organization of endocrine events underlying larval-pupal metamorphosis in the silkworm, Bombyx mori. Journal of Insect Physiology 29, 919-932.

Sakurai, S., Kaya, M., Satake, S., 1998. Hemolymph ecdysteroid titer and ecdysteroid-dependent developmental events in the last-larval stadium of the silkworm, Bombyx mori: role of low ecdysteroid titer in larval pupal metamorphosis and a reappraisal of the head critical period. Journal of Insect Physiology 44, 867-881.

Sakurai, S., Niimi, S., 1997. Developmental changes in juvenile hormone and juvenile hormone acid titers in the hemolymph and in vitro juvenile hormone synthesis by corpora allata of the silkworm, Bombyx mori. Journal of Insect Physiology 43, 875-884.

Schwartz, L. M., 1992. Insect muscle as a model for programmed cell death. 
Journal of Neurobiology 23, 1312-1326.

Sekimoto, T., Iwami, M., Sakurai, S., 2006. Coordinate responses of transcription factors to ecdysone during programmed cell death in the anterior silk gland of the silkworm, Bombyx mori. Insect Molecular Biology 15, 281-292.

Sekimoto, T., Iwami, M., Sakurai, S., 2007. 20-Hydroxyecdysone regulation of two isoforms of the Ets transcription factor E74 gene in programmed cell death in the silkworm anterior silk gland. Insect Molecular Biology 16, 581-590.

Shaffer, B. M., 1963. The isolated Xenopus laevis tail: a preparation for studying the central nervous system and metamorphosis in culture. Journal of embryology and experimental morphology 11, 77-90.

Terashima, J., Yasuhara, N., Iwami, M., Sakurai, S., 2000. Programmed cell death triggered by insect steroid hormone, 20-hydroxyecdysone, in the anterior silk gland of the silkworm, Bombyx mori. Development Genes and Evolution 210, $545-558$.

von Gaudecker, B., Schmale, E. M., 1974. Substrate-histochemical investigations and ultrahistochemocal demonstrations of acid phosphatase in larval and prepupal salivary glands of Drosophila melanogaster. Cell and Tissue Research $155,75-89$.

Wang, Z., Brown, D. D., 1993. Thyroid hormone-induced gene expression program for amphibian tail resorption. The Journal of Biological chemistry 268, 16270-16278. 


\section{Figure Legends}

Figure 1. V3 ASGs underwent PCD in an excess volume of Grace's medium. V3 ASGs were cultured individually in $0.3,3,6$ or $9 \mathrm{ml}$ of medium with $1 \mu \mathrm{M} 20 \mathrm{E}$ for 144 h. (A) The degree of PCD progression was noted with PCD scores as described by Terashima et al. (2000). A score of 0 indicates no change, and a score of 6 indicates the completion of PCD. The PCD score was noted every 24 h. (B-E) The concentration of $\mathrm{H}_{2} \mathrm{O}_{2}$ in medium was measured during the culture of the V3 ASGs in $0.3-9 \mathrm{ml}$ of medium. Each point represents the mean $\pm \mathrm{SD} ; \mathrm{n}=12$ for (A) and 3 for (B-E).

Figure 2. ASGs become competent to respond to 20E on V2. (A) ASGs from IV4 to V7 were incubated in $9 \mathrm{ml}$ of Grace's medium with $1 \mu \mathrm{M} 20 \mathrm{E}$ (+) or without $20 \mathrm{E}$ (-) for $144 \mathrm{~h}$. The ordinate indicates the percentage of the glands that exhibited a given PCD score at the end of the culture. ASGs that scored at or above 4 underwent PCD (Terashima et al., 2000; Iga et al., 2007). Some of the ASGs exhibited the type B form (Kakei et al., 2005), which is indicated with a "B" in the PCD score ( $\mathrm{n}=12$ for each column). (B) The oligonucleosomal ladder first appeared in V2 ASGs when cultured with 20E (V2 +20E). The left column shows a 100-bp DNA fragment ladder. In V1 ASGs, the DNA appears as a smear. (C, c) V1 ASGs after $144 \mathrm{~h}$ of culture in Grace's medium without 20E or (D, d) with 20E. (E, e) V2 ASGs after $144 \mathrm{~h}$ of culture in the medium without $20 \mathrm{E}$ or $(\mathrm{F}, \mathrm{f})$ with $20 \mathrm{E}$. (C-F) Light micrograph and (c-f) DAPI staining show nuclear morphology. Panels $(\mathrm{C}, \mathrm{E}, \mathrm{c}, \mathrm{e})$ and $(\mathrm{F}, \mathrm{f})$ show typical examples of score 0 and score 6 , respectively. Panels (D,d) are typical examples of the type B form. Scale bar, $70 \mu \mathrm{m}$. (G) 
Expression of 20E-responsive genes in V1 and V2 ASGs. ASGs were incubated in $9 \mathrm{ml}$ of the medium with $20 \mathrm{E}$ for $8 \mathrm{~h}$. The genes $E c R$, ecdysone receptor; usp, Ultraspiracle; BR-C , broad complex; BHR3, Bombyx hormone receptor 3; and $R p L 3$, ribosomal protein L3 were selected according to Sekimoto et al. (2006; 2007).

Figure 3. Change in the timing of the death commitment in ASGs. (A) A pair of CA were removed from individual larvae on day 3 of the fourth instar (IV3). Acetone or $0.1 \mu \mathrm{g}$ of JHA was topically applied within hours after the operation. The ASGs of the larvae that successfully molted into the fifth instar were cultured on the same day in $9 \mathrm{ml}$ of Grace's medium with $1 \mu \mathrm{M}$ 20E. (B) Larvae were allatectomized on IV3, and the newly molted V0 larvae were treated with acetone or $0.1 \mu \mathrm{g}$ of JHA. ASGs were obtained on V3 and cultured in $9 \mathrm{ml}$ of the medium with $20 \mathrm{E}$ for $144 \mathrm{~h}$. Ordinates in (A, B) are the same as in Figure 2A ( $\mathrm{n}=12$ for each column in (A) and (B)). (C) Expression of the $K r$-h1 gene in V0, V1 and V2 ASGs is shown in intact larvae (left panel) and in ASGs of the larvae that received the same treatments as those in (B) (right panel).

Figure 4. Involvement of JH in the acquisition of responsiveness to 20E. The CA were removed on IV3, and those larvae received a single application of JHA (0.1 $\mu \mathrm{g} /$ larva) on a given day between V0 and V6. ASGs were obtained two days after JHA application and were cultured in $9 \mathrm{ml}$ of medium with $20 \mathrm{E}$ for $144 \mathrm{~h}$. (A) Responses to 20E as noted with PCD scores (see Fig. 2 for scores) (n=10 for V0-3 and V6; 18 for V4; and 12 for V5. (B) $K r$-h1 expression in ASGs at the time of 
dissection.

Figure 5. ASGs begin to lose their sensitivity to JHA on V5 and completely lose it on V6. Fifth instar larvae were topically treated with acetone (-) or a single dose of JHA (0.1 $\mu \mathrm{g} /$ larva) (+) on the day indicated. ASGs were dissected on V8 irrespective of the day of treatment and cultured in $9 \mathrm{ml}$ of medium with $20 \mathrm{E}$ for $144 \mathrm{~h}$. The ordinate is the same as in Figure 2 ( $\mathrm{n}=12$ for each column).

Figure 6. Schematic representation of a proposed hormonal regulation of the death commitment and the progression of PCD execution in B. mori ASGs. The solid and broken lines indicate ecdysteroids and $\mathrm{JH}$ concentration in the hemolymph, respectively (Satake et al., 1998; Sakurai and Niimi, 1997). 
Fig. 1
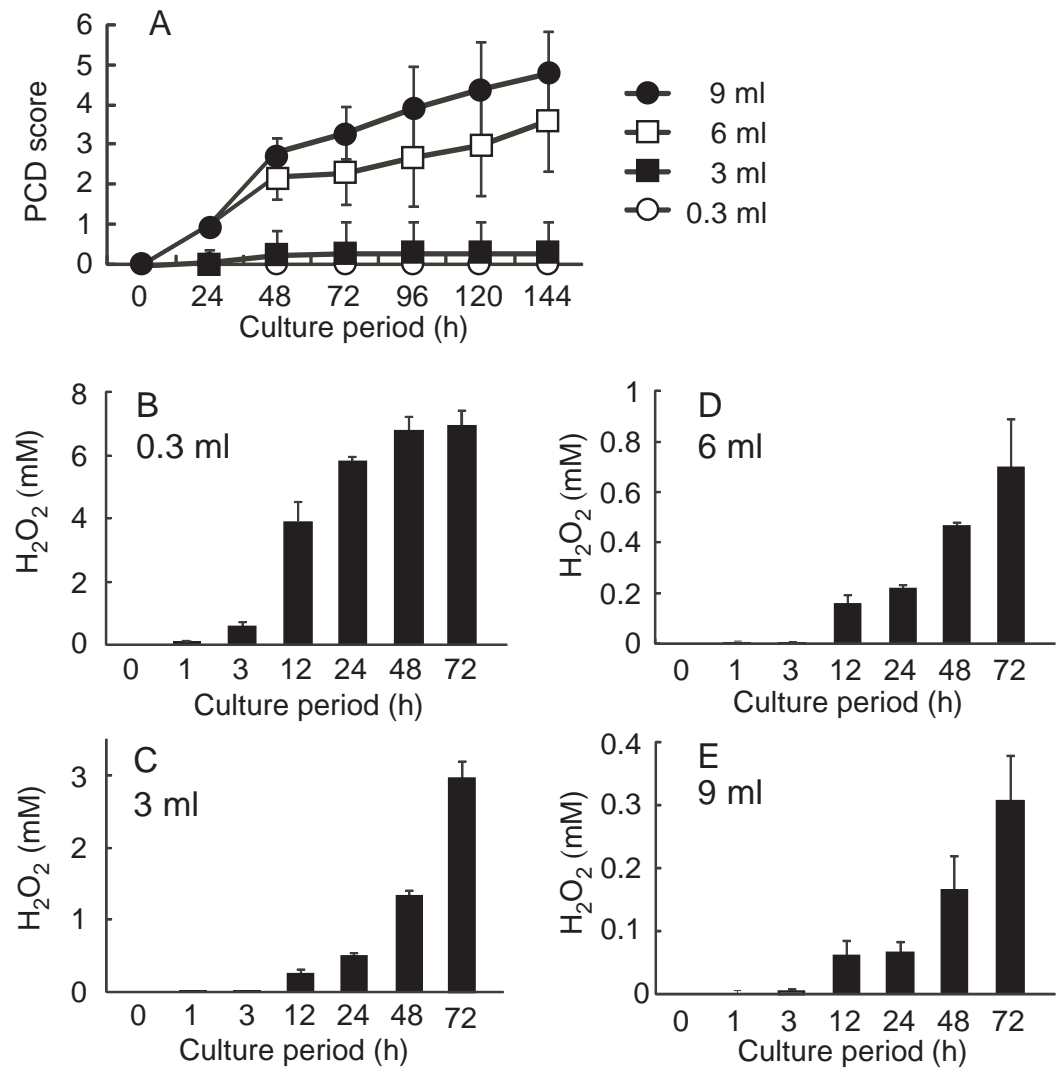
Fig. 2

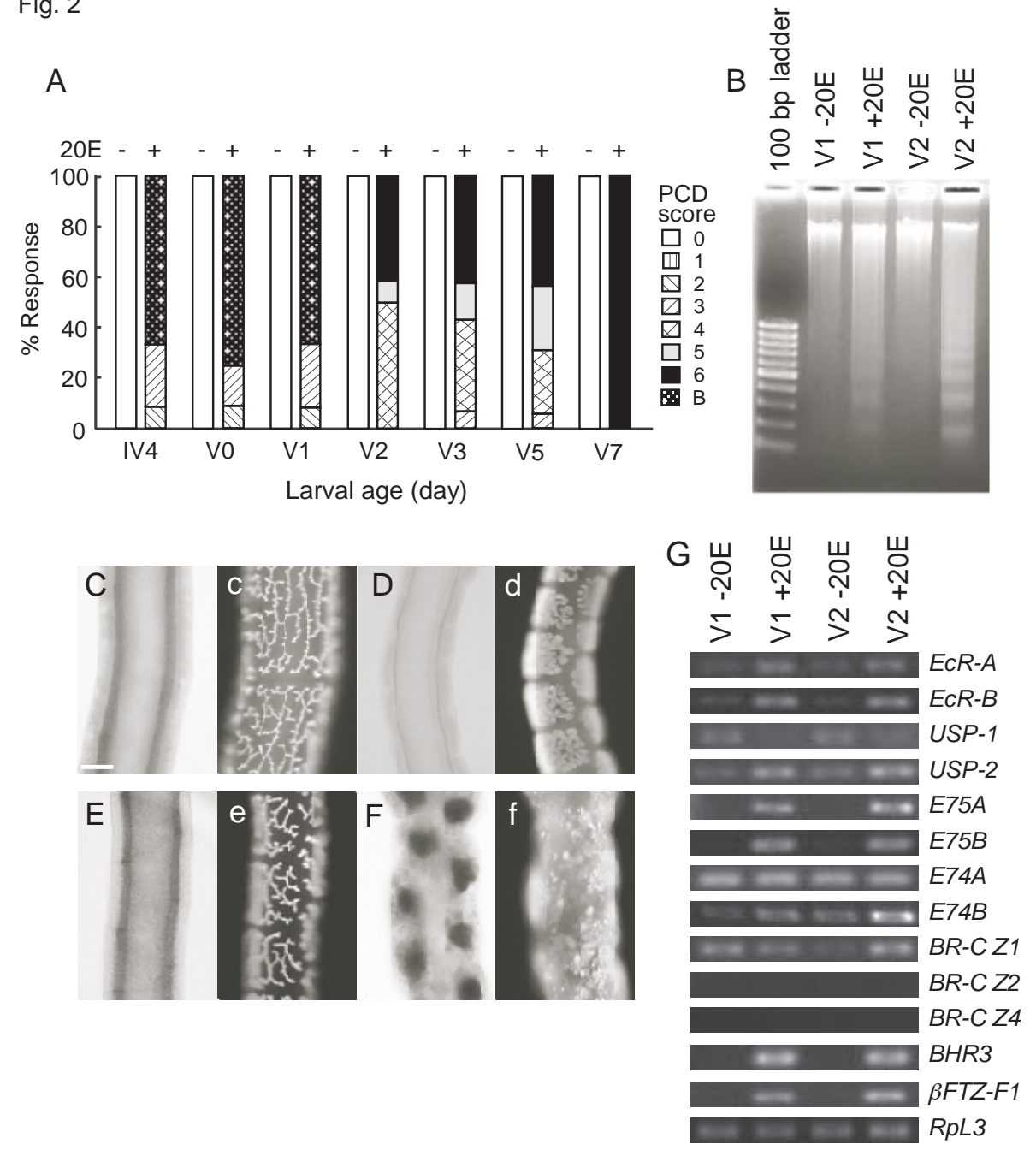


Fig. 3

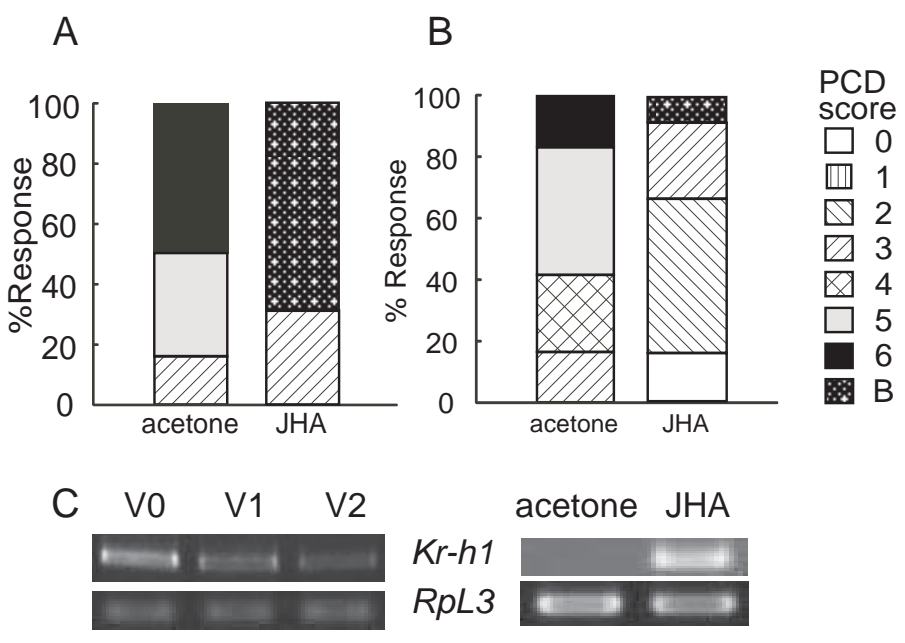


Fig. 4

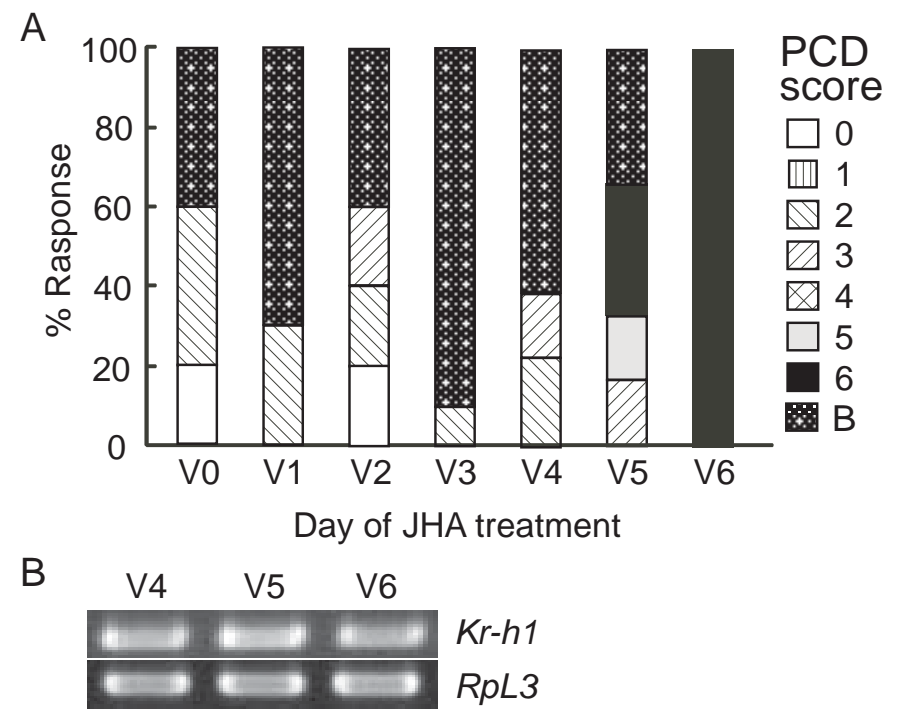


Fig. 5

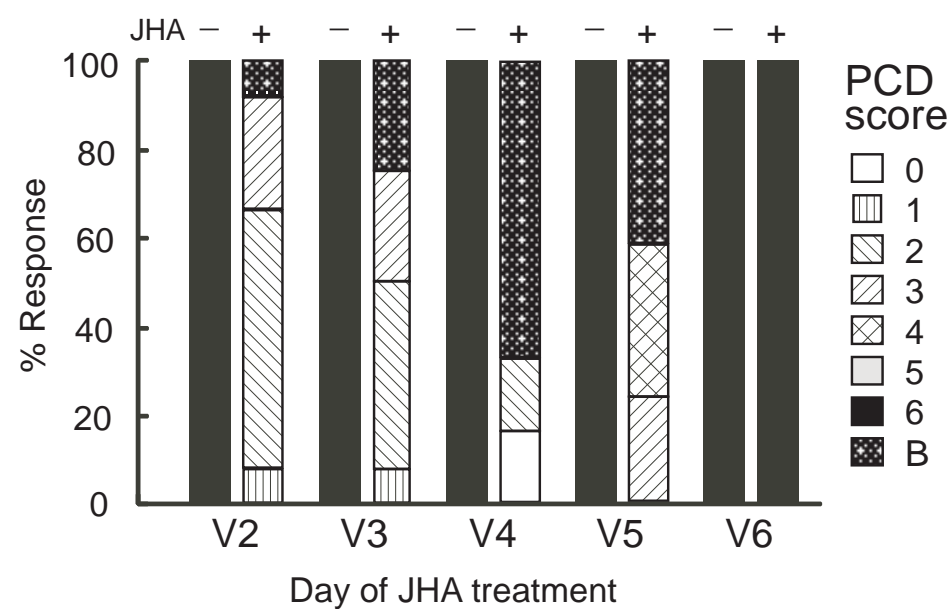

